\title{
Ernst Schütte 65 Jahre
}

\author{
Herrn Prof. Dr. med. Dr. rer. nat. Ernst Schütte \\ zum 65. Geburtstag am 14. 6.1973
}

\begin{abstract}
Sehr verehrter, lieber Herr Schütte!
Im Namen der Mitglieder der Deutschen Gesellschaft für Klinische Chemie möchte ich Ihnen zu Ihrem 65. Geburtstag die herzlichsten Glückwünsche übermitteln.

Die Deutsche Gesellschaft für Klinische Chemie fühlt sich Ihnen in ganz besonderem Maße verbunden:

Durch die Gründung der Zeitschrift für klinische Chemie und klinische Biochemie, durch die Mitbegründung der Gesellschaft und durch ihr stetes Eintreten für das noch junge Fach haben Sie uns in einzigartiger Weise geholfen, die ersten selbständigen Schritte $z u$ tun.

Wir wünschen Ihnen zu Ihrem 65. Geburtstag vor allem Gesundheit und Schaffenskraft für viele weitere Jahre gemeinsamer Arbeit.
\end{abstract}

Deutsche Gesellschaft für Klinische Chemie

J. Büttner

Präsident 